\title{
Space-time symmetries and the Yang-Mills gradient flow
}

\section{Del Debbio, ${ }^{a}$ A. Patella ${ }^{b, c}$ and A. Rago ${ }^{c}$}

${ }^{a}$ Higgs Centre for Theoretical Physics, School of Physics and Astronomy, University of Edinburgh, Edinburgh EH9 3JZ, U.K.

${ }^{b} \mathrm{PH}-\mathrm{TH}, \mathrm{CERN}$,

CH-1211 Geneva 23, Switzerland

${ }^{c}$ School of Computing and Mathematics $\&$ Centre for Mathematical Science, Plymouth University, Plymouth PL4 8AA, U.K.

E-mail: luigi.del.debbio@ed.ac.uk, agostino.patella@plymouth.ac.uk, antonio.rago@plymouth.ac.uk

ABSTRACT: The recent introduction of the gradient flow has provided a new tool to probe the dynamics of quantum field theories. The latest developments have shown how to use the gradient flow for the exploration of symmetries, and the definition of the corresponding renormalized Noether currents. In this paper we introduce infinitesimal translations along the gradient flow for gauge theories, and study the corresponding Ward identities. This approach is readily generalized to the case of gauge theories defined on a lattice, where the regulator breaks translation invariance. The Ward identities in this case lead to a nonperturbative renormalization of the energy-momentum tensor. We discuss an application of this method to the study of dilatations and scale invariance on the lattice.

Keywords: Lattice Gauge Field Theories, Space-Time Symmetries, Renormalization Group 


\section{Contents}

1 Introduction 1

2 Gradient flow - an essential toolkit 3

3 Translations 5

$\begin{array}{ll}3.1 & \text { Probe observables at positive flow time }\end{array}$

4 Translations at positive flow time $r$

$\begin{array}{llr}5 & \text { Dilatations } & 11\end{array}$

6 Space-time symmetries on the lattice 13

$\begin{array}{lll}\text { 6.1 Strategies to renormalize the energy-momentum tensor } & 16\end{array}$

$\begin{array}{lll}7 & \text { Remarks on small flow-time expansion } & 17\end{array}$

$\begin{array}{llr}8 & \text { Conclusions } & 18\end{array}$

$\begin{array}{ll}\text { A Integration of the Lagrange multiplier } & 20\end{array}$

B Large-distance behaviour of the Jacobian matrix 22

\section{Introduction}

The lattice regulator provides a unique framework to investigate non-perturbative properties of non-Abelian gauge theories. However this formulation explicitly breaks the Poincaré group at finite lattice spacing and the exact restoration of the related invariances can be recovered only in the continuum limit.

As space-time symmetries are explicitly broken, the Ward identities associated to translations are violated, and the construction of a renormalized energy-momentum tensor that generates these transformations requires special care. A nonperturbative renormalization of the energy-momentum tensor is necessary in order to guarantee that numerical studies of physical quantities related to the Noether currents are not obscured by lattice artefacts. For instance the study of scale invariance in quantum field theories is a problem that requires the knowledge of a properly-defined energy-momentum tensor.

The lattice energy-momentum tensor can be obtained as a linear combination of all operators with dimension not greater than four allowed by the lattice symmetries. The coefficients have to be tuned in such a way that the Ward identities of the continuum are satisfied up to cutoff effects. This condition guarantees that the operator defined above 
is the generator of the Poincaré transformations in the continuum. This program was articulated in great detail in refs. [1,2].

The approach of [1] is based on the idea that one can probe the lattice energymomentum tensor with a certain number of local observables. However this approach can be used only if the energy-momentum tensor is separated from the probe observables, otherwise extra contact terms due to mixing with higher-dimensional operators might be generated. This problem has been occasionally seen as an intrinsic limitation of the strategy proposed by the authors of [1] (see for instance the introduction of [3], or the works $[4,5]$ in the context of supersymmetry). On the contrary we argue that the limitation originates entirely from the choice of local observables to probe translation symmetry (or any other symmetry indeed).

In this paper we review this program in the light of the recently developed Yang-Mills gradient flow [6-9]. More specifically we use the gradient flow in order to define more appropriate probes for the translation Ward identities. Thanks to its remarkable renormalization properties the gradient flow offers a systematic way to define renormalization-independent observables and finite composite operators. The gradient flow essentially smears the elementary fields on a typical range of order $\sqrt{8 t}$ where $t$ is the flow time. Observables constructed from the fields at some positive flow time are non-local in the elementary fields, and they represent more natural probes for the translation Ward identities. The main goal of this paper is to analyse all possible divergences that can arise from the translation Ward identities on the lattice when observables at positive flow time are used as probes. We shall see that contact terms are completely absent from the Ward identities, and hence they are regular in any space-time point. In section 6 a strategy to renormalize the energy-momentum tensor is proposed. The basic idea to use observables at positive flow time as probes for Ward identities is not new, and has been already applied in ref. [9] to chiral symmetry.

The analysis of divergences and the regularization of Ward identities passes through a complete analysis of the space-time symmetries of the flow equation (section 4), which can be implemented as the equation of motion of a five dimensional theory [8]. Beyond the technical aspects, this analysis also generates new insight.

The Noether current associated to a symmetry is obtained by considering some local version of the symmetry transformation. The Ward identities describe the response to the transformation applied to an ultra-local region of the space-time (a single point, in distributional sense). In the case of translations the Ward identities describe what happens if a single point of space-time gets translated by a certain infinitesimal displacement. If a lattice regulator has being used, this is certainly not the most natural choice. As we shall see in section 4 , the gradient flow provides a natural way to probe symmetries at (any) intermediate length scale, by defining quasi-local transformations, i.e. transformations that modify the fields smoothly within a region with typical linear size of order $\sqrt{8 t}$. These quasi-local transformations do not generate the artificial divergences arising in the ultralocal approach, not even on the lattice.

We extend our analysis to dilatations as well (section 5). We will be able to prove an operatorial version of the Callan-Symanzik equation [10-12], in which the flow time is 
interpreted as a (square) energy scale, and regular expectation values with the insertion of the trace of the energy-momentum tensor source the violation of scale invariance. Our analysis provides a tool to test scale-invariance at all energy scales, which is directly related to the trace anomaly.

In a recent paper [3], the gradient flow is also used to regularize the energy-momentum tensor. This very interesting approach is orthogonal to ours: an operator is defined at each positive flow time $t$ (and it is therefore finite in any regularization scheme), which coincides with the energy-momentum tensor in the $t \rightarrow 0^{+}$limit. This quantity is defined in terms of two coefficients which are calculated in a perturbative expansion. In section 7 we connect our general analysis with the small flow-time expansion, and we outline a possible non-perturbative definition of the coefficients appearing in ref. [3].

We also want to point out that completely different strategies have been recently proposed to renormalize the energy-momentum tensor on the lattice [13, 14].

\section{Gradient flow — an essential toolkit}

In this section we review the definition of the gradient flow, and some of its salient properties that will be relevant for this paper. Throughout this paper we use the notations of ref. [8]. In sections $3,4,5$ and 7 , we focus on the theory on the continuum, regulated using dimensional regularization. The dimension of space-time is taken to be $D=4-2 \epsilon$, but we will not need to use the cutoff explicitly. In section 6 we use an explicit lattice discretization. In the context of lattice gauge theories, the Yang-Mills gradient flow is referred to as Wilson flow (see e.g. [7]), and it has been used in a number of applications [7, 9, 15-19]. However we do not give a review of the Wilson flow here, and we refer the reader to the relevant literature.

Flow equations. The flow of the gauge field $\bar{B}_{t, \mu}(x)$ is defined through the set of equations

$$
\begin{aligned}
\partial_{t} \bar{B}_{t, \mu} & =\bar{D}_{t, \nu} \bar{G}_{t, \nu \mu}, & \left.\bar{B}_{t, \mu}\right|_{t=0} & =A_{\mu} \\
\bar{G}_{t, \nu \mu} & =\partial_{\mu} \bar{B}_{t, \nu}-\partial_{\nu} \bar{B}_{t, \mu}+\left[\bar{B}_{t, \mu}, \bar{B}_{t, \nu}\right], & \bar{D}_{t, \mu} & =\partial_{\mu}+\left[\bar{B}_{t, \mu}, \cdot\right],
\end{aligned}
$$

where the greek indices run only in the $D$-dimensional space, we refer to $t$ as the flow time, and $A_{\mu}(x)=A_{\mu}^{A}(x) T^{A}$ is the fundamental gauge field of the $D$-dimensional theory. Field correlators involving the gauge field at flow time $t$ can be calculated in a local field theory in $D+1$ dimensions, where the field $B_{\mu}(t, x)=B_{\mu}^{A}(t, x) T^{A}$ is a dynamical variable, and a Lagrange multiplier $L_{\mu}(t, x)=L_{\mu}^{A}(t, x) T^{A}$ is introduced to enforce the constraint in eq. (2.1). The bulk action is given by:

$$
S_{\text {bulk }}=-2 \int_{0}^{\infty} d t \int d^{D} x \operatorname{tr} L_{\mu}(t, x)\left\{\partial_{t} B_{\mu}(t, x)-D_{\nu} G_{\nu \mu}(t, x)\right\} .
$$

Integrating out the Lagrange multiplier $L_{\mu}$ yields a delta function in the path integral

$$
\prod_{t, x} \delta\left(B_{\mu}(t, x)-\bar{B}_{t, \mu}(x)\right)
$$


which guarantees that the field $B_{\mu}(t, x)$ at flow time $t$ is the solution of the flow equation $\bar{B}_{t, \mu}(x)$. The generators $T^{A}$ are antihermitean and are normalized as:

$$
\operatorname{tr} T^{A} T^{B}=-\frac{1}{2} \delta_{A B}
$$

A perturbative analysis of the properties of this theory has been discussed in ref. [8]. We just need to report two facts that have been discussed in detail in refs. [7, 8]: (1) the bulk action does not renormalize; (2) composite local operators at positive flow time stay finite when the cutoff is removed, and therefore they do not require renormalization.

Jacobian matrix of the trivializing map. The Jacobian matrix associated with the map between field configurations at two different flow times $\bar{B}_{s} \mapsto \bar{B}_{t}$ (only forward propagation is considered) is:

$$
\bar{J}_{\mu \nu}^{A B}(t, x ; s, y)=\theta(t-s) \frac{\delta \bar{B}_{t, \mu}^{A}(x)}{\delta \bar{B}_{s, \nu}^{B}(y)},
$$

and was already introduced in ref. [6] in the context of the trivializing maps. At leading order in perturbation theory, $\bar{J}$ coincides with the flow propagator, and can be explicitly calculated in coordinate space:

$$
\bar{J}_{\mu \nu}^{A B}(t, x ; s, y)=\delta^{A B} \theta(t-s) \frac{e^{-\frac{|x-y|^{2}}{4(t-s)}}}{[4 \pi(t-s)]^{D / 2}}+\ldots,
$$

where the dots stand for $O\left(g^{2}\right)$ terms and terms that decouple from gauge-invariant observables. ${ }^{1}$ This suggests that, when included in gauge-invariant functions of the fields, the Jacobian matrix decays exponentially. This result can be in fact established at all orders in perturbation theory (see appendix B).

Local gauge-invariance in $\mathbf{D}+\mathbf{1}$ dimensions. The bulk action (2.3) is invariant under gauge transformations that do not depend on the flow time. The bulk action can be made invariant under local gauge transformations in $D+1$ dimensions by introducing a component $B_{0}$ of the gauge field along the flow-time direction. The field-strength tensor is also extended accordingly:

$$
G_{0 \mu}=\partial_{t} B_{\mu}-\partial_{\mu} B_{0}+\left[B_{0}, B_{\mu}\right]
$$

and the bulk action becomes:

$$
S_{\text {bulk }}=-2 \int_{0}^{\infty} d t \int d^{D} x \operatorname{tr} L_{\mu}(t, x)\left\{G_{0 \mu}(t, x)-D_{\nu} G_{\nu \mu}(t, x)\right\} .
$$

The original action in eq. (2.3) is recovered in the $B_{0}=0$ gauge. As the measure in the path integral is invariant under the change of variables that brings to the $B_{0}=0$ gauge, the actions (2.3) and (2.9) describe the same quantum field theory.

\footnotetext{
${ }^{1}$ We would like to thank the Referee of the paper for stressing this point.
} 


\section{Translations}

The action of space-time translations on gauge fields can be defined in a gauge-covariant way $[20,21]$ :

$$
\delta_{\alpha} A_{\mu}(x) \stackrel{\text { def }}{=} \alpha_{\rho}(x) F_{\rho \mu}(x) .
$$

The associated global transformations (i.e. with a uniform $\alpha_{\rho}$ ) reduce to infinitesimal translations up to a field-dependent gauge transformation, and therefore are bona fide translations for any gauge-invariant observable.

The four Noether currents associated with these transformations are gathered in an energy-momentum tensor $T_{\mu \rho}$ that is symmetric and gauge-invariant. This energy-momentum tensor is uniquely defined up to an arbitrary constant times $\delta_{\mu \rho}$ which does not enter into the Ward identities of any space-time symmetry, and therefore it will be set such that $\left\langle T_{\mu \rho}\right\rangle=0$ throughout this paper. For pure Yang-Mills the energy-momentum tensor defined from the gauge-covariant transformations above is:

$$
T_{\mu \rho}=-\frac{2}{g_{0}^{2}}\left\{\operatorname{tr} F_{\sigma \mu} F_{\sigma \rho}-\frac{\delta_{\mu \rho}}{4} \operatorname{tr} F_{\sigma \tau} F_{\sigma \tau}\right\},
$$

and the variation of the action under (3.1) is given by:

$$
\delta_{\alpha} S=\int d^{D} x T_{\mu \rho}(x) \partial_{\mu} \alpha_{\rho}(x) .
$$

The fields are normalized in such a way that the action takes the form:

$$
S=-\frac{1}{2 g_{0}^{2}} \int d^{D} x \operatorname{tr} F_{\sigma \tau} F_{\sigma \tau} .
$$

In particular the action is invariant under the transformation (3.1) when $\alpha_{\rho}$ is chosen to be uniform, i.e. independent of the space-time coordinates. It is important to stress that any explicit breaking of the symmetry generates an extra contribution to $\delta_{\alpha} S$. Such explicit breaking can originate from terms in the action, or from the regularization used to define the theory. For instance the lattice regularization breaks translation symmetry, leading to a non-trivial renormalization of the energy-momentum tensor. We defer the discussion of the broken Ward identities to section 6.

The variation of a generic observable $P$ under the transformation (3.1) can be written as:

$$
\delta_{\alpha} P=\int d^{D} x \alpha_{\rho}(x) \delta_{x, \rho} P \stackrel{\text { def }}{=} \int d^{D} x \alpha_{\rho}(x) \frac{\delta P}{\delta A_{\mu}^{A}(x)} F_{\rho \mu}^{A}(x) .
$$

The corresponding translation Ward identity (TWI) can be written as:

$$
\left\langle\delta_{x, \rho} P\right\rangle=-\left\langle P \partial_{\mu} T_{\mu \rho}(x)\right\rangle .
$$

A more familiar form of the TWI is obtained by choosing for $P$ a product of gauge-invariant local observables; the 1.h.s. of the equation above can be rewritten as the variation of the product of observables, leading to:

$$
\sum_{j} \delta\left(x-x_{j}\right) \frac{\partial}{\partial x_{j}}\left\langle\phi_{1}\left(x_{1}\right) \cdots \phi_{k}\left(x_{k}\right)\right\rangle=-\left\langle\phi_{1}\left(x_{1}\right) \cdots \phi_{k}\left(x_{k}\right) \partial_{\mu} T_{\mu \rho}(x)\right\rangle .
$$


Note that the TWI (3.6) and (3.7) hold for the regulated correlators in the bare theory, because dimensional regularization preserves translation invariance.

A clarification is in order here. When the theory is defined using dimensional regularization and a perturbative expansion, we must address the issue of gauge fixing. Gaugefixing terms and ghost terms are added to the action, and consequently to the energymomentum tensor. However when gauge invariant observables are considered in the TWI, these extra-pieces in the energy momentum tensor do not contribute to the expectation values, so we can safely omit them.

In order to remove the cutoff in equation (3.7), the bare parameters and fields have to be replaced with renormalized ones:

$$
A_{\mu}=Z^{1 / 2} Z_{3}^{1 / 2}\left(A_{\mu}\right)_{R}, \quad g_{0}=\mu^{2 \epsilon} g^{2} Z
$$

and the observables $\phi_{j}$ with their renormalized counterpart $\left(\phi_{j}\right)_{R}$ :

$$
\sum_{j} \delta\left(x-x_{j}\right) \frac{\partial}{\partial x_{j}}\left\langle\phi_{1}\left(x_{1}\right)_{R} \cdots \phi_{k}\left(x_{k}\right)_{R}\right\rangle=-\left\langle\phi_{1}\left(x_{1}\right)_{R} \cdots \phi_{k}\left(x_{k}\right)_{R} \partial_{\mu} T_{\mu \rho}(x)\right\rangle .
$$

Ward identities are a powerful tool to analyse the divergences of Noether currents and related operators. Indeed, the finiteness (in a distributional sense) of the l.h.s. of eq. (3.9) in the $\epsilon \rightarrow 0$ limit, implies the finiteness of the operator $\partial_{\mu} T_{\mu \rho}(x)$. In gauge theories with no scalars this is shown to be equivalent to the finiteness of the energy-momentum tensor itself in the $\epsilon \rightarrow 0$ limit [22-26]. In other words the energy-momentum tensor does not require renormalization in dimensional regularization; in order to avoid the usage of an overabundant notation we will not introduce the symbol $\left(T_{\mu \nu}\right)_{R}$.

For a generic non-local observable $P$, the $\epsilon \rightarrow 0$ limit of both sides of the TWI (3.6) is trickier because contact terms will arise in general, and we will not pursue this direction further. However in the next subsection we will show that, if $P$ is chosen to be an observable that depends on the fields at positive flow-time only, such contact terms do not arise and the corresponding TWI is regular in the $\epsilon \rightarrow 0$ limit.

Let us conclude this introductory discussion by stressing that Ward identities have been used routinely in the context of renormalization. A prominent (and familiar) example of their usage is the renormalization of quark bilinears from chiral Ward identities [27]. Further progress has been made recently in the case of the chiral Ward identities by using probe fields at positive flow time [9]. Following this idea, we will investigate in the following sections the possibility of extending the discussion of TWI at positive flow time.

\subsection{Probe observables at positive flow time}

We want to specialize the TWI (3.6) to the case of a probe observable $P_{T}$ that depends only on the field $\bar{B}_{T, \mu}$ at flow time $T>0$. The variation of $P_{T}$ under the transformation (3.1) can be written using the chain rule:

$$
\delta_{x, \rho} P_{T}=\int d^{D} y \frac{\delta P_{T}}{\delta \bar{B}_{T, \nu}^{B}(y)} J_{\nu \mu}^{B A}(T, y ; 0, x) F_{\rho \mu}^{A}(x),
$$


where the Jacobian matrix defined in eq. (2.6) has been used. Let us emphasise that we are considering here the variation of a probe observable $P_{T}$ induced by an infinitesimal translation of the gauge fields at flow time $t=0$. The expression above is purely algebraic, and it is exact for the regulated theory, i.e. for any value of $\epsilon$. In order to discuss the renormalization of the TWI, the divergence structure of $\delta_{x, \rho} P_{T}$ has to be understood. At first sight, this task seems to be difficult because the Jacobian matrix $J$ is a non-local operator, and has a quite complicated expansion in terms of the elementary fields of the $D$-dimensional theory. However this problem can be completely circumvented by looking at the extended theory in $D+1$ dimensions. Indeed let us consider the composite operator:

$$
\tilde{T}_{0 \rho}(t, x)=-2 \operatorname{tr} L_{\mu}(t, x) G_{\rho \mu}(t, x),
$$

which is defined in the higher-dimensional bulk theory in terms of the Lagrange multiplier $L_{\mu}$ and of the bulk field $G_{\mu \nu}$. Since the Lagrange multiplier appears linearly in the action, any polynomial in $L_{\mu}$ can be explicitly integrated out in the path integral. In particular if the probe observable $P_{T}$ depends only on the field $B_{\mu}(T, x)$ at flow time $T>0$ (and does not depend on the Lagrange multiplier) it is possible to show that:

$$
\left\langle\delta_{x, \rho} P_{T}\right\rangle=\left\langle P_{T} \tilde{T}_{0 \rho}(0, x)\right\rangle .
$$

The calculation is rather technical and is reported in appendix A. As for the case discussed above, this equation holds for any value of $\epsilon$. Let us choose now the particular form $P_{T}=$ $\phi_{1, T}\left(x_{1}\right) \cdots \phi_{k, T}\left(x_{k}\right)$, where $\phi_{i, T}\left(x_{i}\right)$ is a local observable in the field $B_{\mu}(T, x)$, i.e. depends only on $B_{\mu}$ and its derivatives at flow time $T$ and in the space-time point $x_{i}$. Using eq. (3.12) the problem of identifying the divergences of $\left\langle\delta_{x, \rho} P_{T}\right\rangle$ is reduced to the standard task of identifying the divergences of the product of local operators in the $(D+1)$-dimensional theory. We know already that local operators in the bulk do not require renormalization (notice in particular that some of the $x_{i}$ 's can coincide, and still no singular contact terms are generated). The operator $\tilde{T}_{0 \rho}(0, x)$ is located on the boundary and therefore has to be renormalized. In principle $\tilde{T}_{0 \rho}(0, x)$ could mix with any other gauge-invariant operator of dimension 5 that transforms as a vector under Lorentz transformations. However the Wick contractions involving the Lagrange multiplier $L_{\mu}$ are such that the two-point correlation functions of $\tilde{T}_{0 \rho}(0, x)$ and any local operator composed from the gauge field at flow time zero vanish up to contact terms. Divergent additive renormalizations to $\tilde{T}_{0 \rho}(0, x)$ by such operators are therefore excluded. Divergences could arise from the mixing with operators involving the Lagrange multiplier, but $\tilde{T}_{0 \rho}(0, x)$ itself is the only one with dimension not greater than 5 and the required symmetry properties. Therefore the operator $\tilde{T}_{0 \rho}(0, x)$ can renormalize only multiplicatively. We anticipate here that this argument does not rely on using dimensional regularization, and holds also on the lattice. Finally we notice that no extra divergences arise when $x$ hits one of the $x_{i}$ 's as the operators $\tilde{T}_{0 \rho}(0, x)$ and $\phi_{i, T}\left(x_{i}\right)$ are always in separate points in the $(D+1)$-dimensional space.

In dimensional regularization, since translation invariance is preserved, one can combine eq. (3.12) and the TWI (3.6) into:

$$
\left\langle P_{T} \tilde{T}_{0 \rho}(0, x)\right\rangle=-\left\langle P_{T} \partial_{\mu} T_{\mu \rho}(x)\right\rangle,
$$


which shows that $\tilde{T}_{0 \rho}$ stays finite in the $\epsilon \rightarrow 0$ limit, and does not require to be renormalized. Thanks to eq. (3.12) the same conclusion holds for the expectation value $\left\langle\delta_{x, \rho} P_{T}\right\rangle$. This essentially means that the differential operator $\delta_{x, \rho}$ can at most generate a multiplicative renormalization when applied to an observable $P_{T}$ which is a function of fields at positive flow time only, but no contact terms are generated. However the multiplicative renormalization factor is constrained to be equal to one in dimensional regularization thanks to translation invariance. In section 6 we will see how this discussion generalizes to the case of a regularization that breaks translation invariance, such as the lattice.

\section{Translations at positive flow time}

The flow equations are invariant under global translations. This means that one is free to translate the fields at any flow time $t$, the result on any observable will be exactly the same as one would obtain by first translating the boundary fields and then evolving them up to flow time $t$. This argument can be taken one step further, by generalizing the local transformation (3.1) as:

$$
\bar{\delta}_{t, \alpha} P=\int d^{D} x \alpha_{\rho}(x) \bar{\delta}_{t, x, \rho} P=\int d^{D} x \alpha_{\rho}(x) \frac{\delta P}{\delta \bar{B}_{t, \mu}^{A}(x)} \bar{G}_{t, \rho \mu}^{A}(x) .
$$

This equation defines a family of transformations parametrized by the flow time $t$. Clearly for $t=0$ the usual translation defined in the previous section is recovered. The differential operator $\bar{\delta}_{t, x, \rho}$ depends only on the fields $\bar{B}_{t, \mu}$ at the space-time point $x$, but is not local in the fundamental field $A_{\mu}$. As a consequence, the finite transformation generated by $\bar{\delta}_{t, x, \rho}$ modifies the fundamental field $A_{\mu}$ not only at $x$, but in a neighborhood of it. This neighborhood has a typical linear size of order $\sqrt{8 t}$.

In close analogy to eq. (3.12), it is possible to show that:

$$
\left\langle\bar{\delta}_{t, x, \rho} P_{T}\right\rangle=\left\langle P_{T} \tilde{T}_{0 \rho}(t, x)\right\rangle .
$$

Note that in this case the tensor $\tilde{T}$ is evaluated at flow time $t$, while it was computed on the boundary in eq. (3.12).

If $\alpha_{\rho}$ is uniform, the transformation generated by $\bar{\delta}_{t, x, \rho}$ reduces to the composition of a canonical infinitesimal translation of the field $\bar{B}_{t, \mu}$ and a field-dependent gauge transformation, which is immaterial when acting on gauge-invariant observables. Since the flow equations are invariant under global translations, $\bar{\delta}_{t, \alpha}$ reduces to a canonical infinitesimal translation of the fields at any flow time when acting on gauge-invariant observables:

$$
\int d^{D} y \bar{\delta}_{t, y, \rho} \phi(x)=\partial_{\rho} \phi(x) .
$$

It is interesting to consider some special instances of eq. (4.3), e.g. by choosing an observable $\phi_{T}(x)$ that depends only on the field $\bar{B}_{\mu}$ and its derivative at flow time $T$ and space-time position $x$. If $T=t$ then a local version of eq. (4.3) holds:

$$
\bar{\delta}_{t, y, \rho} \phi_{t}(x)=\delta(y-x) \partial_{\rho} \phi_{t}(x) .
$$


If $T>t$ then the delta function gets regularized and a milder result holds. If $V$ is a sphere centered in $x$ with radius $r$ then roughly speaking:

$$
\int_{V} d^{D} y \bar{\delta}_{t, y, \rho} \phi_{T}(x)=\partial_{\rho} \phi_{T}(x)+O\left(e^{-\frac{r^{2}}{4(T-t)}}\right) .
$$

We refer to the end of this section for the proof of a precise version of this equation.

The nice feature of the differential operator $\bar{\delta}_{t, \alpha}$ for $t>0$ is that it depends only on fields at positive flow time, and therefore it does not require renormalization in any regularization scheme. Associated with it, for each flow time $t$, there is a new energy-momentum tensor and a new TWI. As the transformation (4.1) is non-local in the original field $A_{\mu}$, this new energy-momentum tensor is not local in the $D$-dimensional theory. However it is possible to write it in terms of local operators in the $(D+1)$-dimensional theory by exploiting the space-time symmetries of the $(D+1)$-dimensional theory.

The bulk action in eq. (2.9) is clearly invariant under $(D+1)$-dimensional canonical translations (the translation in the flow time is broken only by boundary effects). Following the procedure described on the boundary, infinitesimal local translations can be upgraded to the following gauge-covariant transformations acting on the bulk fields:

$$
\left\{\begin{array}{l}
\delta_{\alpha} B_{M}(t, x) \stackrel{\text { def }}{=} \alpha_{R}(t, x) G_{R M}(t, x), \\
\delta_{\alpha} L_{\mu}(t, x) \stackrel{\text { def }}{=} \alpha_{R}(t, x) D_{R} L_{\mu}(t, x),
\end{array}\right.
$$

with the constraint that $\alpha_{0}(0, x)=0$. Capital indices run from 0 to $D$, and the index 0 denotes the flow time. We will always consider here observables that do not depend on the Lagrange multiplier $L_{\mu}$. The variation of one of these observables $P$ is:

$$
\begin{aligned}
\delta_{\alpha} P & =\int_{0}^{\infty} d t \int d^{D} x \alpha_{R}(t, x) \delta_{t, x, R} P \stackrel{\text { def }}{=} \\
& \stackrel{\text { def }}{=} \int_{0}^{\infty} d t \int d^{D} x \alpha_{R}(t, x) \frac{\delta P}{\delta B_{M}^{A}(t, x)} G_{R M}^{A}(t, x) .
\end{aligned}
$$

The variation of the bulk action under the transformation (4.6) defines a $(D+1)$ dimensional energy-momentum tensor:

$$
\begin{aligned}
\delta_{\alpha} S_{\mathrm{bulk}} & =\int_{0}^{\infty} d t \int d^{D} x \tilde{T}_{M R}(t, x) \partial_{M} \alpha_{R}(t, x) \\
\tilde{T}_{0 R} & =-2 \operatorname{tr} L_{\mu} G_{R \mu}, \\
\tilde{T}_{\nu R} & =2 \operatorname{tr} L_{\mu} D_{\nu} G_{R \mu}-2 \operatorname{tr} D_{\nu} L_{\mu} G_{R \mu}-2 \operatorname{tr} D_{\mu} L_{\mu} G_{\nu R}+2 \delta_{R 0} \operatorname{tr} L_{\nu} D_{\mu} G_{\mu 0},
\end{aligned}
$$

up to terms that are proportional to the constraint and therefore vanish in expectation values. Notice that the operator $\tilde{T}_{0 R}$ for $R \neq 0$ is the same that appears in eqs. (3.12) and (3.13). As the number of differential operators is proliferating, we find convenient to review at this point the meaning of all of them. The differential operator $\bar{\delta}_{t, x, \rho}$ acts on fields that satify already the flow equation. The fields are deformed at flow time $t$, and the flow equation propagates this deformation to all other flow times. To make sense of this picture, we use the fact that the flow is invertible at least at finite cutoff. In particular the 
operator $\delta_{x, \rho}=\bar{\delta}_{0, x, \rho}$ deforms the fields on the boundary, i.e. the initial condition for the flow equation, and therefore the deformation is propagated to any positive flow time. The operator $\delta_{t, x, \rho}$ that we have just defined is completely different, as it acts on the $(D+1)$ dimensional fields before the flow equation is imposed. It deforms the fields locally in the $(D+1)$-dimensional space and such deformation is not propagated in flow time. Of course if one starts with a field configuration that satisfies the flow equation, its deformation will in general not satisfy the same equation. The variation in the equation is reabsorbed by the deformation of the Lagrange multiplier.

For any $t>0$, the Ward identities associated with the transformation (4.6) are:

$$
\left\langle\delta_{t, x, \rho} P\right\rangle=-\left\langle P \partial_{M} \tilde{T}_{M R}(t, x)\right\rangle .
$$

For a probe observable $P_{T}$ that depends only on the field $B_{\mu}$ at flow time $T>t$, the l.h.s. of the previous equation vanishes. In this particular case, eq. (4.11) can be written as:

$$
\left\langle P_{T} \partial_{t} \tilde{T}_{0 R}(t, x)\right\rangle=-\left\langle P_{T} \partial_{\mu} \tilde{T}_{\mu R}(t, x)\right\rangle .
$$

We will not need to consider the case $R=0$ in this section, and we will therefore develop the arguments below for the case where $R=\rho$ spans the usual space-time directions. We will see now how eq. (4.12) leads to the Ward identities for the family of transformations defined in eq. (4.1), and how one can use this equation to prove eq. (4.5). Note that all fields that are computed at flow time $t>0$ have finite correlators, and do not require renormalization as the regularization is removed.

We would like to integrate eq. (4.12) in flow time in an interval $(0, t)$. However this equation is valid only at positive flow time. The problem is that for $t=0$ the Ward identity (4.11) gets an extra contribution from the fact that the boundary fields are transformed along with the bulk ones. Moreover eq. (4.12) is valid for bare fields at finite cutoff. At positive $t$, since only fields in the bulk are involved, this equation does not have any divergences and its $\epsilon \rightarrow 0$ limit can be safely taken. Therefore, after the cutoff is removed, we integrate eq. (4.12) in an interval $\left(t_{0}, t\right)$ first with $0<t_{0}<t<T$ :

$$
\left\langle P_{T} \tilde{T}_{0 \rho}(t, x)\right\rangle=\left\langle P_{T} \tilde{T}_{0 \rho}\left(t_{0}, x\right)\right\rangle-\left\langle P_{T} \partial_{\mu} \int_{t_{0}}^{t} d s \tilde{T}_{\mu \rho}(s, x)\right\rangle
$$

and then we take the $t_{0} \rightarrow 0^{+}$limit. We have already proven eq. (4.2):

$$
\left\langle P_{T} \tilde{T}_{0 \rho}(t, x)\right\rangle=\left\langle\bar{\delta}_{t, x, \rho} P_{T}\right\rangle,
$$

and eq. (3.13):

$$
\lim _{t_{0} \rightarrow 0^{+}}\left\langle P_{T} \tilde{T}_{0 \rho}\left(t_{0}, x\right)\right\rangle=-\left\langle P_{T} \partial_{\mu} T_{\mu \rho}(x)\right\rangle .
$$

By using these results, eq. (4.13) can be repackaged into the TWI associated with the differential operator $\bar{\delta}_{t, x, \rho}$, which defines the corresponding energy momentum tensor $\bar{T}_{\mu \rho}$ :

$$
\begin{aligned}
\left\langle\bar{\delta}_{t, x, \rho} P_{T}\right\rangle & =-\left\langle P_{T} \partial_{\mu} \bar{T}_{\mu \rho}(t, x)\right\rangle, \\
\bar{T}_{\mu \rho}(t, x) & =T_{\mu \rho}(x)+\int_{0}^{t} d s \tilde{T}_{\mu \rho}(s, x) .
\end{aligned}
$$


Clearly this TWI reduces to eq. (3.6) for $t=0$; however these manipulations are meaningful only if the integral appearing in the energy-momentum tensor (4.17) is finite.

The possible divergences of $\tilde{T}_{\mu \rho}(s, x)$ at $s \rightarrow 0^{+}$are classified in terms of all operators of dimension up to 6 that can mix with $\tilde{T}_{\mu \rho}(s, x)$. Such operators must contain at least a Lagrange multiplier, i.e. an operator of dimension 3. Therefore, by taking into account the Lorentz structure, $\tilde{T}_{\mu \rho}(s, x)$ can mix with operators of dimension 6 and 4 . However it is easy to see that gauge-invariance excludes operators of dimension 4 . This means that $\tilde{T}_{\mu \rho}(s, x)$ has at most a logarithmic divergence for $s \rightarrow 0^{+}$, which is integrable. This concludes our discussion, as the singularity in the energy-momentum tensor (4.17) is integrable.

In order to understand the action of the operator $\bar{\delta}_{t, x, \rho}$ on fields defined at $T>t$, let us now integrate eq. (4.12) in flow time in the interval $(t, T)$. Using eq. (4.2) again:

$$
\left\langle\bar{\delta}_{t, x, \rho} P_{T}\right\rangle=\left\langle\bar{\delta}_{T, x, \rho} P_{T}\right\rangle+\left\langle P_{T} \partial_{\mu} \int_{t}^{T} d s \tilde{T}_{\mu \rho}(s, x)\right\rangle .
$$

Let us consider a local observable $\phi(T, x)$ at positive flow time $T$, and let $X_{T}$ be a product of other local observables at the same flow time $T$ but different space-time positions. We choose $P_{T}=X_{T} \phi(T, x)$ in the previous equation and integrate it on a space-time sphere $V$ with radius $r$ and centered in $x$. Assuming that all the local observables in $X_{T}$ lie outside of the sphere $V$, and by using eq. (4.4), one gets:

$$
\left\langle X_{T} \int_{V} d^{D} y \bar{\delta}_{t, y, \rho} \phi(T, x)\right\rangle=\left\langle X_{T} \partial_{\rho} \phi(T, x)\right\rangle+\left\langle X_{T} \phi(T, x) \int_{t}^{T} d s \int_{\partial V} d S_{\mu} \tilde{T}_{\mu \rho}(s, x)\right\rangle .
$$

The operator $\tilde{T}_{\mu \rho}$ contains only terms that are linear in the Lagrange multiplier $L_{\mu}$. Since the propagator $L B$ is exponentially suppressed with the space-time separation of the two fields, the contribution of the last term of the previous equation is exponentially suppressed if all the fields are far enough from the boundary $\partial V$ of the sphere. If $\bar{r}$ is distance from $\partial V$ of the closest operator (clearly $\bar{r} \leq r$ ), then:

$$
\left\langle X_{T} \int_{V} d^{D} y \bar{\delta}_{t, y, \rho} \phi(T, x)\right\rangle=\left\langle X_{T} \partial_{\rho} \phi(T, x)\right\rangle+O\left(e^{-\frac{\bar{r}^{2}}{4(T-t)}}\right)
$$

which is the precise form of eq. (4.5).

\section{Dilatations}

In order to discuss dilatations, we are going to extend the definition of the differential operator $\bar{\delta}_{t, x, \rho}$ in eq. (4.1) to include the flow-time direction:

$$
\bar{\delta}_{t, x, R} P \stackrel{\text { def }}{=} \frac{\delta P}{\delta \bar{B}_{t, \mu}^{A}(x)} \bar{G}_{t, R \mu}^{A}(x),
$$

where $R$ runs over all $D+1$ dimensions. This differential operator can be related to the $\tilde{T}_{0 R}$ operator at generic flow time:

$$
\left\langle\bar{\delta}_{t, x, R} P_{T}\right\rangle=\left\langle P_{T} \tilde{T}_{0 R}(t, x)\right\rangle,
$$

by integrating explicitly the Lagrange multiplier, as shown in appendix A. 
Local dilatations are a special case of local translations. On the boundary a local dilatation is generated by the transformation (3.1) with $\alpha_{\rho}(x)=x_{\rho} \beta(x)$. A global dilatation corresponds to a uniform $\beta$. The flow equation is also invariant under dilatations provided that the flow time is rescaled too by its classical dimension. Local dilatations in the bulk are generated by the transformation (4.6) with $\alpha_{\rho}(t, x)=x_{\rho} \beta(t, x)$ and $\alpha_{0}(t, x)=2 t \beta(t, x)$.

In practice we consider the equation:

$$
\begin{aligned}
& \left\langle P_{T} \partial_{t}\left[2 t \tilde{T}_{00}(t, x)+x_{\rho} \tilde{T}_{0 \rho}(t, x)\right]\right\rangle= \\
& \quad\left\langle P_{T}\left[2 \tilde{T}_{00}(t, x)+\tilde{T}_{\mu \mu}(t, x)\right]\right\rangle-\left\langle P_{T} \partial_{\mu}\left[2 t \tilde{T}_{\mu 0}(t, x)+x_{\rho} \tilde{T}_{\mu \rho}(t, x)\right]\right\rangle,
\end{aligned}
$$

which follows trivially from eq. (4.12) and stays finite in the $\epsilon \rightarrow 0$ limit. It is interesting to notice that the operator $2 \tilde{T}_{00}+\tilde{T}_{\mu \mu}$ (which is almost the trace of the bulk energymomentum tensor, except that different components are weighted with the dimension of the corresponding coordinate) might break dilatation invariance in the bulk. However some trivial algebra shows that this generalized trace is a divergence:

$$
2 \tilde{T}_{00}(t, x)+\tilde{T}_{\mu \mu}(t, x)=\partial_{\mu} \tilde{T}_{0 \mu}(t, x),
$$

up to terms that are proportional to the constraint generating the flow equation, which we can omit as they vanish in expectation values. This result is not surprising as the flow equation is invariant under dilatations. We plug this result back into eq. (5.3), and integrate it in the flow-time interval $(0, t)$ with $t<T$, following closely what we have done already for the TWI:

$$
\begin{aligned}
& \left\langle P_{T}\left[2 t \tilde{T}_{00}(t, x)+x_{\rho} \tilde{T}_{0 \rho}(t, x)\right]\right\rangle-\lim _{t_{0} \rightarrow 0^{+}}\left\langle P_{T}\left[2 t_{0} \tilde{T}_{00}\left(t_{0}, x\right)+x_{\rho} \tilde{T}_{0 \rho}\left(t_{0}, x\right)\right]\right\rangle= \\
& -\left\langle P_{T} \partial_{\mu} \int_{0}^{t} d s\left[2 s \tilde{T}_{\mu 0}(s, x)+x_{\rho} \tilde{T}_{\mu \rho}(s, x)-\tilde{T}_{0 \mu}(s, x)\right]\right\rangle .
\end{aligned}
$$

Now we can use eqs. (5.2) and (3.13), together with the observation that:

$$
\lim _{t_{0} \rightarrow 0^{+}}\left\langle P_{T} t_{0} \tilde{T}_{00}\left(t_{0}, x\right)\right\rangle=0
$$

as $\tilde{T}_{00}\left(t_{0}, x\right)$ diverges at most logarithmically, and we can repackage eq. (5.5) into the dilatation Ward identity (DWI):

$$
\begin{aligned}
\left\langle\left\{2 t \bar{\delta}_{t, x, 0}+x_{\rho} \bar{\delta}_{t, x, \rho}\right\} P_{T}\right\rangle & =-\left\langle P_{T} \partial_{\mu} \bar{D}_{\mu}(t, x)\right\rangle+\left\langle P_{T} T_{\mu \mu}(x)\right\rangle, \\
\bar{D}_{\mu}(t, x) & =x_{\rho} T_{\mu \rho}(x)+\int_{0}^{t} d s \tilde{D}_{\mu}(s, x), \\
\tilde{D}_{\mu} & =2 t \tilde{T}_{\mu 0}+x_{\rho} \tilde{T}_{\mu \rho}-\tilde{T}_{0 \mu}
\end{aligned}
$$

where $2 t \bar{\delta}_{t, x, 0}+x_{\rho} \bar{\delta}_{t, x, \rho}$ is the differential operator that generates dilatations at flow time $t$. Usual power-counting arguments show that the integral in the dilatation current $\bar{D}_{\mu}$ is finite. As usual $P_{T}$ is an observable that depends on the field $B_{\mu}$ at flow time $T$ only, and $T>t$. Of course dilatations are not symmetries of pure Yang-Mills. The trace of the 
energy-momentum tensor that appears in the r.h.s. of the DWI (5.7) is the source of the anomaly.

If $\phi_{T}(x)$ is an observable that depends only on the field $\bar{B}_{T}$ at flow time $T$ and spacetime point $x$, then the global dilatation is simply:

$$
\int d^{D} y\left\{2 t \bar{\delta}_{t, y, 0}+y_{\rho} \bar{\delta}_{t, y, \rho}\right\} \phi_{T}(x)=\left\{2 T \frac{d}{d T}+x_{\rho} \partial_{\rho}+d_{\phi}\right\} \phi_{T}(x),
$$

where $d_{\phi}$ is the dimension of the operator $\phi_{T}$. The DWI for $\phi_{T}$ reduces to a very simple form:

$$
\left(2 T \frac{d}{d T}+d_{\phi}\right)\left\langle\phi_{T}\right\rangle=\left\langle\phi_{T} \int d^{D} x T_{\mu \mu}(x)\right\rangle_{c} .
$$

This equation is the operatorial form of the Callan-Symanzik equation [10-12], in which $(8 T)^{-1 / 2}$ is the energy scale, and contact terms are absent. Equation (5.11) is extremely interesting as it allows the trace of the energy-momentum tensor to be probed just by looking at the evolution under gradient flow of observables.

\section{Space-time symmetries on the lattice}

If the lattice regulator is used, then the explicit breaking of translation symmetry generates an extra term in the TWI (3.6), which implies that the energy-momentum tensor will require renormalization. Even after subtracting the divergences, the TWI (3.6) is valid in this case only up to terms that vanish in infinite-cutoff limit. We will review how this happens, following the presentation in ref. [1].

At finite lattice spacing $a$, a regularized version of the transformation (3.1), can be defined by choosing for example a particular discretization of the field strength $F_{\mu \nu}$ (for definiteness one can adopt the clover plaquette definition) and by replacing the fundamental field $A_{\mu}(x)$ with the link variable $U_{\mu}(x)$. The discretized transformation will be denoted by $\hat{\delta}$ :

$$
\begin{aligned}
\hat{\delta}_{\alpha} U_{\mu}(x) & =\alpha_{\rho}(x) \hat{F}_{\rho \mu}(x) U_{\mu}(x), \\
\hat{\delta}_{x, \rho} P & =\frac{1}{a^{3}} \hat{F}_{\rho \mu}^{A}(x) \partial_{U_{\mu}(x)}^{A} P,
\end{aligned}
$$

where $\partial_{U_{\mu}(x)}^{A}$ is the left Lie derivative on the gauge group with respect to $U_{\mu}(x)$. This transformation leaves the measure of the path integral unchanged, however it is not a symmetry as the lattice action $\hat{S}$ is not invariant when the parameter $\alpha_{\rho}$ is chosen to be uniform:

$$
\hat{\delta}_{\alpha} \hat{S}=-\sum_{x} a^{4} \alpha_{\rho}(x)\left\{R_{\rho}(x)+\hat{\partial}_{\mu} \hat{T}_{\mu \rho}^{(1)}(x)\right\}
$$

where $\hat{T}_{\mu \rho}^{(1)}$ is your favourite naively-discretized energy-momentum tensor. The $R_{\rho}$ operator, which depends on the choice of discretization for $\hat{T}_{\mu \rho}^{(1)}$, is the residual term in the Ward identity, and comes from the explicit breaking of the symmetry. It is a higher-dimensional operator, and vanishes in the formal $a \rightarrow 0$ limit (i.e. on fixed field configurations that have a smooth continuum limit). However formally subleading corrections cannot be neglected 
in field correlators as subleading coefficients can combine with divergent expectation values giving rise to finite contributions. By standard dimensional analysis arguments one can isolate the possible divergences in $R_{\rho}$ :

$$
R_{\rho}=\frac{1}{Z_{\delta}} \bar{R}_{\rho}+\left(\frac{c_{1}}{Z_{\delta}}-1\right) \hat{\partial}_{\mu} \hat{T}_{\mu \rho}^{(1)}+\frac{c_{2}}{Z_{\delta}} \hat{\partial}_{\mu} \hat{T}_{\mu \rho}^{(2)}+\frac{c_{3}}{Z_{\delta}} \hat{\partial}_{\mu} \hat{T}_{\mu \rho}^{(3)}
$$

where $\bar{R}_{\rho}$ is a finite operator, and the $\hat{T}_{\mu \rho}^{(2,3)}$ operators are:

$$
\begin{aligned}
& \hat{T}_{\mu \rho}^{(2)}=\delta_{\mu \rho} \sum_{\sigma \tau} \operatorname{tr} \hat{F}_{\sigma \tau} \hat{F}_{\sigma \tau}, \\
& \hat{T}_{\mu \rho}^{(3)}=\delta_{\mu \rho} \sum_{\sigma} \operatorname{tr} \hat{F}_{\mu \sigma} \hat{F}_{\mu \sigma} .
\end{aligned}
$$

If the renormalized energy-momentum tensor on the lattice is defined as:

$$
\left(\hat{T}_{\mu \rho}\right)_{R}=\sum_{i} c_{i}\left\{\hat{T}_{\mu \rho}^{(i)}-\left\langle\hat{T}_{\mu \rho}^{(i)}\right\rangle\right\}
$$

the Ward identity associated with the transformation (6.2) becomes:

$$
\left\langle Z_{\delta} \hat{\delta}_{x, \rho} P+P \bar{R}_{\rho}(x)\right\rangle=-\left\langle P \hat{\partial}_{\mu} \hat{T}_{\mu \rho}(x)_{R}\right\rangle .
$$

As for the case of dimensional regularization, we will discuss the continuum limit of this equation for two possible choices of the observable $P$ : a product of local observables, or a generic observable that depends on the fields at positive flow time only.

Let us choose for $P$ a product of properly renormalized local observables at separate points. The assumption that translation symmetry has to be recovered in the continuum limit implies that the coefficients $c_{i}$ and $Z_{\delta}$ can be tuned in such a way that:

1. The energy-momentum tensor is finite in the continuum limit, i.e. the following limit:

$$
\lim _{a \rightarrow 0}\left\langle\hat{\phi}_{1}\left(x_{1}\right)_{R} \cdots \hat{\phi}_{k}\left(x_{k}\right)_{R} \hat{T}_{\mu \rho}(x)_{R}\right\rangle=\left\langle\phi_{1}\left(x_{1}\right)_{R} \cdots \phi_{k}\left(x_{k}\right)_{R} T_{\mu \rho}(x)\right\rangle
$$

is finite up to contact terms.

2. The local Ward identity is finite in the continuum limit, provided that fields are located in separated positions:

$$
\lim _{a \rightarrow 0}\left\langle\hat{\phi}_{1}\left(x_{1}\right)_{R} \cdots \hat{\phi}_{k}\left(x_{k}\right)_{R} \hat{\partial}_{\mu} \hat{T}_{\mu \rho}(x)_{R}\right\rangle=0 .
$$

In particular this implies that the term $\left\langle\bar{R}_{\rho}(x) \hat{\phi}_{1}\left(x_{1}\right)_{R} \cdots \hat{\phi}_{k}\left(x_{k}\right)_{R}\right\rangle$ is zero in the continuum limit up to contact terms.

3. The l.h.s. of eq. (6.8) is finite in the continuum limit once integrated over some volume $V$, and is equal to:

$$
\lim _{a \rightarrow 0} \sum_{x \in V} a^{4}\left\langle\left\{Z_{\delta} \hat{\delta}_{x, \rho}+\bar{R}_{\rho}(x)\right\} \hat{\phi}_{1}\left(x_{1}\right)_{R} \cdots \hat{\phi}_{k}\left(x_{k}\right)_{R}\right\rangle=\sum_{x_{j} \in V} \frac{\partial}{\partial x_{j}}\left\langle\phi_{1}\left(x_{1}\right)_{R} \cdots \phi_{k}\left(x_{k}\right)_{R}\right\rangle,
$$


under the condition that the $\phi_{i}$ 's are separated from the volume boundary by some physical distance. Two type of contact terms appear when $x$ hits some of the fields in the l.h.s. of the previous equation. Some of these contact terms diverge like $a^{-4}$ and generate the correct nonzero result in the continuum limit. Other contact terms match analogous contact terms arising from the r.h.s. of eq. (6.8), have a zero average and do not contribute to the integral. This second type of contact terms that plagues the local Ward identity shows that local operators do not necessarily represent the most natural choice to probe the translation Ward identity.

Let us consider now a probe observable $\hat{P}_{T}$ which is a function of the fields at positive flow time $T$ only. As in dimensional regularization, the lattice differential operator $\hat{\delta}_{x, \rho}$ can be represented by a local operator in the $(D+1)$-dimensional theory. As discussed in appendix A, the following equality holds at any lattice spacing:

$$
\left\langle\hat{\delta}_{x, \rho} \hat{P}_{T}\right\rangle=-2\left\langle\hat{P}_{T} \operatorname{tr} L_{\mu}(0, x) \hat{F}_{\rho \mu}(x)\right\rangle,
$$

which is the discretized version of eq. (3.12). As discussed in section 4 the operator $\operatorname{tr} L_{\mu}(0, x) \hat{F}_{\rho \mu}(x)$ renormalizes multiplicatively. One can therefore introduce the renormalized operator:

$$
\tilde{T}_{0 \rho}(0, x)_{R}=-2 Z_{\delta} \operatorname{tr} L_{\mu}(0, x) \hat{F}_{\rho \mu}(x),
$$

such that the limits that appear in the following chain of equations are finite:

$$
\lim _{a \rightarrow 0}\left\langle\hat{P}_{T} \tilde{T}_{0 \rho}(0, x)_{R}\right\rangle=\lim _{a \rightarrow 0} Z_{\delta}\left\langle\hat{\delta}_{x, \rho} \hat{P}_{T}\right\rangle=\left\langle\delta_{x, \rho} P_{T}\right\rangle .
$$

As the operator $\tilde{T}_{0 \rho}(0, x)$ is renormalization group invariant in the continuum, the renormalization of the corresponding lattice-discretized operator is finite, i.e. $Z_{\delta}$ is depends on the lattice spacing only through the bare coupling. This finite normalization must be fixed by requiring that the continuum differential operator $\delta_{x, \rho}$ defined through eq. (6.14) generates translations, or in other words satisfies eq. (4.5) for $t=0$. It is important to notice also that no contact term is generated in eq. (6.14), therefore its continuum limit is regular as a function of the space-time position. Roughly speaking, through eq. (6.14) the use of observables at positive flow time allows the renormalization of the differential operator $\hat{\delta}_{x, \rho}$ without using the assumption that translation invariance must be recovered in the continuum limit. Under such supplementary assumption one concludes that the coefficients $c_{i}$ can be tuned in such a way that: (1) the energy-momentum tensor is finite in the continuum limit, i.e. the following limit:

$$
\lim _{a \rightarrow 0}\left\langle\hat{P}_{T} \hat{T}_{\mu \rho}(x)_{R}\right\rangle=\left\langle P_{T} T_{\mu \rho}(x)\right\rangle
$$

is finite and regular at the space-time point $x$ (as no contact terms are generated); (2) the contribution of the operator $\bar{R}$ vanishes in the continuum limit at any space-time point $x$ :

$$
\lim _{a \rightarrow 0}\left\langle\hat{P}_{T} \bar{R}_{\rho}(x)\right\rangle=0 .
$$

Putting all together, up to subleading corrections in the lattice spacing:

$$
\left\langle\delta_{x, \rho} P_{T}\right\rangle=Z_{\delta}\left\langle\hat{\delta}_{x, \rho} \hat{P}_{T}\right\rangle=-\left\langle\hat{P}_{T} \hat{\partial}_{\mu} \hat{T}_{\mu \rho}(x)_{R}\right\rangle .
$$


Probe observables defined in terms of the fields at some positive flow time generate neither delta functions in the Ward identity nor contact terms, and seem to represent a more natural choice to probe translation symmetry (or any other symmetry).

\subsection{Strategies to renormalize the energy-momentum tensor}

Let us consider a local observable $\phi_{t, \rho}(x)$ in the fields at positive flow time $t$. For reasons that will be clear soon, we choose it to transform like a vector with respect to the hypercubic symmetry. Up to subleading corrections in the lattice spacing, eq. (6.17) implies:

$$
Z_{\delta}\left\langle\hat{\delta}_{x, \rho} \phi_{t, \rho}(0)\right\rangle=-\sum_{i} c_{i}\left\langle\phi_{t, \rho}(0) \hat{\partial}_{\mu} \hat{T}_{\mu \rho}^{(i)}(x)\right\rangle .
$$

In the continuum limit this equation is valid for any space-time position $x$ (even $x=0$ ), any flow time $t$, and any probe observable. The ratios $c_{i} / Z_{\delta}$ are therefore highly constrained by this equation. We have chosen a vector probe so that the expectation values in eq. (6.18) do not vanish at $x=0$.

We need to fix now the multiplicative renormalization $Z_{\delta}$. This can be done in several ways. For instance one can enforce eq. (4.20) within a two-point function. One can consider the wall average of a local observable $\phi_{t}(x)$ :

$$
\Phi_{t}\left(x_{4}\right)=\frac{1}{L^{3}} \sum_{\mathbf{x}} a^{3} \phi_{t}\left(\mathbf{x}, x_{4}\right)
$$

and choose the integration volume in eq. (4.20) to be the space-time slice $-d<x_{4}<d$ :

$$
Z_{\delta}\left\langle\Phi_{t}\left(z_{4}\right) \sum_{y_{4}=-d}^{d} \sum_{\mathbf{y}} a^{4} \hat{\delta}_{y, 4} \Phi_{t}(0)\right\rangle=\left\langle\Phi_{t}\left(z_{4}\right) \hat{\partial}_{4} \Phi_{t}(0)\right\rangle+O\left(e^{-\frac{\bar{r}^{2}}{4 t}}\right) .
$$

The operator $\Phi_{t}\left(z_{4}\right)$ must lay outside of the integration slice. The distance $\bar{r}$ that controls the exponential is the minimum between $d$ and $\left|z_{4}-d\right|$. In order to suppress the exponential correction, $\bar{r}$ has to be larger than the smearing range $\sqrt{8 t}$. If the exponential is negligible, then there is a range of values of $d$ for which the l.h.s. of eq. (6.20) is constant, and this can be easily checked in a numerical calculation.

An alternative method, based on the same idea, consists in using a Schrödinger functional setup, with boundaries at $x_{4}= \pm L_{4}$. One needs to engineer boundary conditions such that the background field $F_{\mu \nu}$ depends on the coordinate $x_{4}$. In this case eq. (4.20) becomes:

$$
Z_{\delta}\left\langle\sum_{y_{4}=-d}^{d} \sum_{\mathbf{y}} a^{4} \hat{\delta}_{y, 4} \Phi_{t}(0)\right\rangle_{S F}=\left\langle\hat{\partial}_{4} \Phi_{t}(0)\right\rangle_{S F}+O\left(e^{-\frac{\bar{r}^{2}}{4 t}}\right) .
$$

The distance $\bar{r}$ that controls the exponential is the minimum between $d$ and $L_{4}-d$. The advantage of this approach is that only 1-point functions need to be considered.

Finally one can decide to fix the multiplicative renormalization by means of the DWI (5.11). However one has to take into account corrections coming from the compact geometry of the space-time in lattice simulations:

$$
\left(2 t \frac{d}{d t}+d_{\phi}\right)\left\langle\phi_{t}\right\rangle=\left(c_{2}+\frac{c_{3}}{4}\right)\left\langle\phi_{t}(0) \sum_{x_{i}=-d}^{d} a^{4} T_{\mu \mu}^{(2)}(x)\right\rangle_{c}+O\left(e^{-\frac{\bar{r}^{2}}{4 t}}\right) .
$$

The distance $\bar{r}$ that controls the exponential is the minimum between $d$ and $L-d$. 


\section{Remarks on small flow-time expansion}

As already shown in ref. [3], one can obtain the energy momentum tensor from the small flow-time expansion of the following two operators:

$$
\begin{aligned}
Y_{\mu \rho}(t, x) & =-2\left\{\operatorname{tr} G_{\sigma \mu}(t, x) G_{\sigma \rho}(t, x)-\frac{\delta_{\mu \rho}}{4} \operatorname{tr} G_{\sigma \tau}(t, x) G_{\sigma \tau}(t, x)\right\}, \\
E(t, x) & =-\frac{1}{2} \operatorname{tr} G_{\sigma \tau}(t, x) G_{\sigma \tau}(t, x) .
\end{aligned}
$$

The small flow-time expansions of the operators $E(t, x)$ and $Y_{\mu \rho}(t, x)$ are organized in terms of the dimension $d_{k}$ of the possible mixing renormalized boundary operators $\Theta_{R}^{(k)}$ :

$$
\begin{aligned}
Y_{\mu \rho}(t, x) & =\alpha_{Y}(t)\left[T_{\mu \rho}(x)-\frac{\delta_{\mu \rho}}{4} T_{\sigma \sigma}(x)\right]+\sum_{d_{Y, k} \geq 6} t^{d_{Y, k} / 2-2} c_{Y, k}(t ; \mu) \Theta_{R, \mu \nu}^{(Y, k)}(x ; \mu), \\
E(t, x) & =\langle E(t, x)\rangle+\alpha_{E}(t) T_{\mu \mu}(x)+\sum_{d_{E, k} \geq 6} t^{d_{E, k} / 2-2} c_{E, k}(t ; \mu) \Theta_{R}^{(E, k)}(x ; \mu) .
\end{aligned}
$$

The coefficients $\alpha_{Y}(t)$ and $\alpha_{E}(t)$ are renormalization group invariant, and have a pertubative expansion in terms of the running coupling $g(q)$ at the energy scale $q=(8 t)^{-1 / 2}$. As calculated in ref. [3]:

$$
\begin{aligned}
& \alpha_{Y}(t)=g_{M S}^{2}(q)+2 b_{0}\left[\ln \sqrt{\pi}+\frac{7}{16}\right] g_{M S}^{4}(q)+O\left(g_{M S}^{6}(q)\right), \\
& \alpha_{E}(t)=\frac{1}{2 b_{0}}+\left(\frac{109}{176}-\frac{b_{1}}{2 b_{0}^{2}}\right) g_{M S}^{2}(q)+O\left(g_{M S}^{4}(q)\right) .
\end{aligned}
$$

The calculation is done in the MS renormalization scheme. $b_{0}$ and $b_{1}$ are the coefficients of the expansion of the beta function:

$$
\beta(g)=-b_{0} g^{3}-b_{1} g^{5}+O\left(g^{7}\right) .
$$

The small flow-time behaviour of the coefficients $c_{k}(t ; \mu)$ is dictated by the renormalization group equation. These coefficients are at most logarithmically divergent. ${ }^{2}$

We propose here a strategy to determine the coefficients $\alpha_{Y}(t)$ and $\alpha_{E}(t)$ nonperturbatively up to $O(t)$ corrections. Let us focus on the trace of the energy momentum for now.

\footnotetext{
${ }^{2}$ The coefficients $c_{k}$ are dimensionless and depend on the running coupling constant $g(\mu)$ and on the ratio $q / \mu$. They satisfy a renormalization-group equation:

$$
\begin{aligned}
{\left[\delta^{j k} q \frac{\partial}{\partial q}-\delta^{j k} \beta(g) \frac{\partial}{\partial g}-\gamma^{j k}(g)\right] c_{j} } & =0, \\
\gamma^{j k}(g) & =\gamma_{0}^{j k} g^{2}+O\left(g^{4}\right),
\end{aligned}
$$

where the anomalous dimension matrix accounts for mixing of operators under renormalization group. The leading behaviour of $c_{k}(t ; \mu)$ at large $q$ (i.e. small $t$ ) is governed by the leading term of the anomalous dimension at small $g$ :

$$
c_{k}(t ; \mu) \simeq\left[g(q)^{-\gamma_{0} / b_{0}}\right]_{k j} v_{j}
$$

for some vector $v$ that depends on the initial condition of the renormalization group equation. This shows that $c_{k}(t ; \mu)$ can have at most a logarithmic divergence in $t$.
} 
Given a probe observable $\phi_{T}$ at positive flow time $T$, one can define an effective coefficient $\alpha_{E}^{\text {eff }}(t)$ by imposing that only the leading term of OPE contributes to $E(t, x)$ :

$$
\left\langle\phi_{T} \int d^{D} x E(t, x)\right\rangle_{c}=\alpha_{E}^{\mathrm{eff}}(t)\left\langle\phi_{T} \int d^{D} x T_{\mu \mu}(x)\right\rangle .
$$

Notice that $\alpha_{E}^{\text {eff }}(t)$ defined in this way depends on the probe observable. By using eq. (7.4), one easily sees that:

$$
\alpha_{E}^{\mathrm{eff}}(t)=\alpha_{E}(t)+O(t)
$$

therefore the nonuniversal terms are at least $O(t)$. Here $O(t)$ has to be interpreted up to logarithmic corrections. By using the dilatation Ward identity in the form of eq. (5.11), one obtains the explicit representation:

$$
\alpha_{E}^{\mathrm{eff}}(t)\left(2 T \frac{d}{d T}+d_{\phi}\right)\left\langle\phi_{T}\right\rangle=\left\langle\phi_{T} \int d^{D} x E(t, x)\right\rangle_{c}
$$

In a region in which the nonuniversal $O(t)$ contribution are small, then the following operatorial relation holds:

$$
T_{\mu \mu}(x)=\frac{1}{\alpha_{E}^{\mathrm{eff}}(t)}[E(t, x)-\langle E(t, x)\rangle]+O(t) .
$$

It is worth to stress that this nonperturbative definition of $\alpha_{E}^{\text {eff }}(t)$ provides a definition of the trace of the energy-momentum tensor that is correct up to order $t$ (times logarithms), while a perturbative definition of $\alpha_{E}^{\mathrm{eff}}(t)$ as it is pursued in ref. [3] would give rise or errors that are of order $\ln ^{-2 n} t$.

Analogously one can define an effective coefficient $\alpha_{Y}^{\text {eff }}(t)$ by imposing the integrated TWI, for instance in the form:

$$
\left\langle\phi_{T}(x) \phi_{T}(0) \int d^{D-1} \mathbf{y}\left[Y_{D k}(t, \mathbf{y}, d)-Y_{D k}(t, \mathbf{y},-d)\right]\right\rangle+O\left(e^{-\frac{\bar{r}^{2}}{4 T}}\right)=\alpha_{Y}^{\mathrm{eff}}(t)\left\langle\phi_{T}(x) \partial_{k} \phi_{T}(0)\right\rangle .
$$

In this formula we have separated space and time coordinates $x=\left(\mathbf{x}, x_{D}\right)$. The local TWI (4.16) has been integrated in a space-time slice $-d<x_{D}<d$. The index $k$ runs from 1 to $D-1$, the point $x$ lays outside of the integration volume, and $\bar{r}$ is the minimum between $d,\left|x_{D}+d\right|$ and $\left|x_{D}-d\right|$. Also in this case, by using eq. (7.3), one sees that:

$$
\begin{aligned}
\alpha_{Y}^{\mathrm{eff}}(t) & =\alpha_{Y}(t)+O(t), \\
T_{\mu \rho}(x)-\frac{\delta_{\mu \rho}}{4} T_{\sigma \sigma}(x) & =\frac{1}{\alpha_{Y}^{\mathrm{eff}}(t)} Y_{\mu \rho}(t, x)+O(t) .
\end{aligned}
$$

\section{Conclusions}

Since the renormalization properties of the gradient flow have been clarified, the latter provides a theoretically robust way to investigate the dynamics of gauge theories, and several interesting applications have already appeared since it was originally introduced. In this paper we focus on the possibility of using the gradient flow for studying space-time 
symmetries like translations and dilatations. An important corollary of our study is that the gradient flow can be used to define a properly-normalized energy-momentum tensor for pure Yang-Mills theories defined on a lattice.

The main idea used in this work, inspired by the study in ref. [9], is that the variations under infinitesimal local translations of correlators of fields along the flow can be used to generate translation Ward identities, which encode the symmetry properties of the quantum field theory. We have explored two applications of this idea.

First we studied the case where the transformation is defined on the fields at flow time $t=0$, and we obtained the Ward identities using probe operators at positive flow time. The divergencies of the correlators appearing in these identities have been analysed using a representation of the gradient flow in terms of a $(D+1)$-dimensional local field theory. When a lattice regulator is used, translation symmetry is broken by the regulator, and the energy-momentum tensor undergoes renormalization. A finite energy-momentum tensor can only be defined after the subtraction of divergent mixings with other operators. The Ward identities for the renormalized lattice energy-momentum tensor using probe operators at time $T>0$ are shown in eq. (6.17); the key feature is that these identities can be used to fix the renormalization coefficients in a nonperturbative way. These results extend the programme that was first laid out in refs. [1] to the case of probe operators smeared using the gradient flow. Numerical simulations are needed to verify that this is a viable method in practice; they are deferred to future investigations.

Because the gradient flow commutes with uniform translations, we can also study the Ward identities obtained by transforming the fields at nonvanishing flow time $t$. Once again a $(D+1)$-dimensional representation of the gradient flow allows us to analyse the structure of the field correlators in terms of local fields in the bulk. We have obtained the renormalized Ward identities that are generated by these transformations. They are universal properties of the field correlators, reflecting the translation invariance of the physical world, and do not depend on the regulator used to define the bare theory. The Noether currents appearing in these Ward identities are related to the energy-momentum tensor of the original $D$-dimensional theory in eq. (4.16).

Our analysis includes the case of dilatations. Indeed local dilatations are a special case of local translations. Studying local dilatations in the bulk, we were able to write dilatation Ward identities for operators at generic flow time $T$. These Ward identities show explicitly the anomalous breaking of scale invariance, and thereby provide a new tool to study the trace of the energy-momentum tensor. The variation of the probe fields along the gradient flow is directly related to the correlator of the trace of the energy-momentum tensor with the probe fields, as shown in eq. (5.11). This is a remarkable result that allows the scale invariance of the theory to be probed using the gradient flow.

An interesting extension of the results in ref. [3] emerges naturally in the framework used here to discuss the transformation properties under dilatations. In ref. [3] the $D$ dimensional energy-momentum tensor was defined using a perturbative determination of the small flow-time expansion of operators defined in the bulk. It is possible to introduce a nonperturbative definition of the leading coefficients in this expansion, and to compute them making use of probe observables along the gradient flow, see e.g. eq. (7.13). 
Using the gradient flow to study space-time symmetries is a fertile research direction. The recent extension of the gradient flow to theories with fermions [9] should enable a straightforward generalization of our arguments to gauge theories coupled to matter. We plan to come back on these topics in future studies.

\section{Acknowledgments}

We are indebted to Martin Lüscher for insightful comments at all stages of this work. AP would like to thank him for enlightening discussions and constant inspiration. We would like to thank Roman Zwicky for critical discussions of the manuscript. Part of this work has been discussed and developed during the "Strongly interacting dynamics beyond the Standard Model and the Higgs boson" hosted by the Higgs Centre for Theoretical Physics in Edinburgh, U.K..

\section{A Integration of the Lagrange multiplier}

When observables depend linearly on the Lagrange multiplier $L_{\mu}$, like in the case of the $\tilde{T}_{M R}$ defined in eqs. (4.9) and (4.10), the field $L_{\mu}$ can be explicitly integrated out. In order to do so, it is convenient to introduce the kernel $J(t, x ; s, y)$ that satisfies the linearized flow equation:

$$
\begin{aligned}
\partial_{t} J_{\mu \nu}^{A B}(t, x ; s, y) & =\mathcal{D}_{x ; t, \mu \rho}^{A C} J_{\rho \nu}^{C B}(t, x ; s, y), \\
\mathcal{D}_{\mu \nu} \delta B_{\nu} & \stackrel{\text { def }}{=}\left[G_{\mu \nu}, \delta B_{\nu}\right]+D_{\nu} D_{\nu} \delta B_{\mu}-D_{\nu} D_{\mu} \delta B_{\nu},
\end{aligned}
$$

for any $t>s$, with conditions:

$$
\left\{\begin{array}{l}
J_{\mu \nu}^{A B}\left(t^{+}, x ; t, y\right)=\delta_{A B} \delta_{\mu \nu} \delta(x-y) \\
J_{\mu \nu}^{A B}(t, x ; s, y)=0 \text { for } t<s .
\end{array}\right.
$$

The Jacobian matrix in eq. (2.6) satisfies the same set of equations provided that the field $B$ satisfies the flow equation:

$$
\bar{J}_{\nu \mu}^{B A}(t, y ; s, x)=\theta(t-s) \frac{\delta \bar{B}_{t, \nu}^{B}(y)}{\delta \bar{B}_{s, \mu}^{A}(x)}=\left.J_{\nu \mu}^{B A}(t, y ; s, x)\right|_{B=\bar{B}} .
$$

Let us consider now the operator:

$$
L_{\mu}^{A}(t, x) X_{\mu}^{A}(t, x),
$$

where $X(t, x)$ is a local function of the field $B$ and its derivatives, and let $P$ be a gaugeinvariant operator that depends on the field $B$ only. The insertion of the operator $L X$ is generated by the following change of variable in the path integral:

$$
\left(B^{\prime}\right)_{\mu}^{A}(t, x)=B_{\mu}^{A}(t, x)+\int_{0}^{t} d s \int d^{D} y J_{\mu \nu}^{A B}(t, x ; s, y) \alpha(s, y) X_{\nu}^{B}(s, y),
$$


where $\alpha$ is a generic infinitesimal function that vanishes on the boundary, introduced for convenience of calculation. The Jacobian determinant of this transformation can be readily calculated:

$$
\begin{aligned}
\operatorname{det} \frac{\delta B^{\prime}}{\delta B} & =1+\theta(0) \delta^{D}(0) \int_{0}^{\infty} d t d s \int d^{D} x d^{D} y \alpha(s, y) \frac{\delta X_{\mu}^{A}(s, y)}{\delta B_{\mu}^{A}(t, x)} \\
& \stackrel{\text { def }}{=} 1+\int_{0}^{\infty} d t \int d^{D} x \alpha(t, x) \mathcal{X}(t, x)
\end{aligned}
$$

where $\mathcal{X}$ is a local function of $B$ (and its derivatives). ${ }^{3}$ In order to make precise sense of this formal expression one needs to introduce a regulator. In dimensional regularization $\delta^{D}(0)$ is regularized to zero and $\mathcal{X}$ vanishes. We will comment on the lattice regularization later.

Assuming $\mathcal{X}=0$, when the proposed change of variable is applied to the expectation value of $P\left[B^{\prime}\right]$ one easily gets the following algebraic relation:

$$
\langle\delta P\rangle=\langle P \delta S\rangle,
$$

where the variation of the action produces the insertion of the operator $L X$ :

$$
\delta S=\int_{0}^{\infty} d t \int d^{D} x \alpha(t, y) L_{\mu}^{A}(t, x) X_{\mu}^{A}(t, x) .
$$

Finally one can expand the variation of $P$ in eq. (A.10), plug the variation of the action in, and use the arbitrariness of $\alpha$ to obtain the wanted result:

$$
\left\langle\int_{t}^{\infty} d s \int d^{D} y \frac{\delta P}{\delta B_{\mu}^{A}(s, y)} J_{\mu \nu}^{A B}(s, y ; t, x) X_{\nu}^{B}(t, x)\right\rangle=\left\langle P L_{\mu}^{A}(t, x) X_{\mu}^{A}(t, x)\right\rangle .
$$

This equation shows that, if $P$ depends on the field $B$ at flow time $s$ smaller than $t$ only, then $\left\langle P L_{\mu}^{A}(t, x) X_{\mu}^{A}(t, x)\right\rangle$ vanishes. From a perturbative point of view this can be understood by noticing that the field $L$ propagates into a field $B$ only forward, and the contractions of $L$ with fields $B$ inside $X$ vanish in dimensional regularization. If a regularization (e.g. the lattice) that generates a nonvanishing $\mathcal{X}$ is used then this property of flow-time ordering is spoiled at fixed cutoff, and the definition of the composite operator $L B$ requires some care. An easy way out is to define the $L B$ operator by means of the following limit:

$$
\left[L_{\mu}^{A} X_{\mu}^{A}\right](t, x)=\lim _{\epsilon \rightarrow 0^{+}} L_{\mu}^{A}(t, x) X_{\mu}^{A}(t-\epsilon, x),
$$

where the $\epsilon \rightarrow 0^{+}$limit has to be taken after the cutoff is removed. The insertion of this operator is generated by the change of variable:

$$
\left(B^{\prime}\right)_{\mu}^{A}(t, x)=B_{\mu}^{A}(t, x)+\int_{0}^{t-\epsilon} d s \int d^{D} y J_{\mu \nu}^{A B}(t, x ; s, y) \alpha(s, y) X_{\nu}^{B}(s, y),
$$

\footnotetext{
${ }^{3}$ We are using here that:$$
\frac{\delta J_{\mu \nu}^{A B}(t, x ; s, y)}{\delta B^{A}(t, x)}=0 .
$$

This is correct if the flow-time direction is discretized on a lattice, and the flow-time derivative in the action is discretized as a forward derivative (as it is done in [9]). If a more general regularization is used, then the discussion becomes more complicated and we will not pursue this direction here.
} 
(up to terms that vanish with $\epsilon$ ). The Jacobian determinant is exactly one in this case in any regularization, ${ }^{4}$ and the previous calculation can be replicated exactly by yielding eq. (A.12) again, where the correct flow-time ordering has to be understood.

\section{B Large-distance behaviour of the Jacobian matrix}

In order to dump the gauge degrees of freedom of the field it is useful to modify the flow equation like:

$$
\partial_{t} B_{\mu}=D_{\nu} G_{\nu \mu}+\alpha_{0} D_{\mu} \partial_{\nu} B_{\nu}
$$

As discussed in ref. [8], gauge invariant observables do not depend on $\alpha_{0}$. The Jacobian matrix $J(t, x ; s, y)$ satisfies the linearized flow equation:

$$
\begin{aligned}
\partial_{t} J_{\mu \nu}^{A B}(t, x ; s, y) & =\mathcal{D}_{x ; t, \mu \rho}^{A C} J_{\rho \nu}^{C B}(t, x ; s, y), \\
\mathcal{D}_{\mu \nu} \delta B_{\nu} & \stackrel{\text { def }}{=}\left[G_{\mu \nu}, \delta B_{\nu}\right]+D_{\nu} D_{\nu} \delta B_{\mu}-D_{\nu} D_{\mu} \delta B_{\nu}+\alpha_{0} D_{\mu} \partial_{\nu} \delta B_{\nu}-\alpha_{0}\left[\partial_{\nu} B_{\nu}, \delta B_{\mu}\right],
\end{aligned}
$$

for any $t>s$, with conditions:

$$
\left\{\begin{array}{l}
J_{\mu \nu}^{A B}\left(t^{+}, x ; t, y\right)=\delta_{A B} \delta_{\mu \nu} \delta(x-y) \\
J_{\mu \nu}^{A B}(t, x ; s, y)=0 \text { for } t<s
\end{array}\right.
$$

The operator $\mathcal{D}$ can be conveniently split into a free part and an $O\left(g_{0}\right)$ reminder:

$$
\mathcal{D}_{\mu \nu} \stackrel{\text { def }}{=} \delta_{\mu \nu} \partial^{2}+\left(\alpha_{0}-1\right) \partial_{\nu} \partial_{\mu}+\mathcal{R}_{\mu \nu}
$$

The linearized flow equation can be written also in the following integral form:

$$
\begin{aligned}
J_{\mu \nu}^{A B}(t, x ; s, y)= & \delta^{A B} K_{t-s ; \mu \nu}(x-y) \\
& +\int_{s}^{t} d \sigma \int d^{D} z K_{t-\sigma ; \mu \lambda}(x-z) \mathcal{R}_{\sigma, z ; \lambda \rho}^{A C} J_{\rho \nu}^{C B}(\sigma, z ; s, y),
\end{aligned}
$$

with the following definition of the free kernel:

$$
K_{t ; \mu \nu}(x)=\int \frac{d^{D} p}{(2 \pi)^{D}} \frac{e^{i p x}}{p^{2}}\left\{\left(\delta_{\mu \nu} p^{2}-p_{\mu} p_{\nu}\right) e^{-t p^{2}}+p_{\mu} p_{\nu} e^{-\alpha_{0} t p^{2}}\right\}
$$

The recursive solution of the integral equation (B.6) provides the perturbative expansion of the Jacobian matrix.

\footnotetext{
${ }^{4}$ When a lattice regulator is used, the change of variable becomes:

$$
V_{\mu}^{\prime}(t, x)=\left[1+\int_{0}^{t-\epsilon} d s \sum_{y} a^{4} J_{\mu \nu}^{A B}(t, x ; s, y) \alpha(s, y) X_{\nu}^{B}(s, y)\right] V_{\mu}(t, x),
$$

where $V_{\mu}(t, x)$ is the link variable at flow time $t$, and the Jacobian matrix is defined by the discretized version of eqs. (A.1) and (A.2). In particular the operator $\mathcal{D}$ is obtained by differentiating the Wilson flow equation $[6,7]$.
} 
Gauge-invariant observables do not depend on the parameter $\alpha_{0}$, and one can conveniently choose $\alpha_{0}=1$. With this choice the free kernel becomes simply:

$$
K_{t ; \mu \nu}(x)=\delta_{\mu \nu} \frac{e^{-\frac{|x|^{2}}{4 t}}}{(4 \pi t)^{D / 2}} .
$$

The generic term of the iterative solution of the integral equation (B.6) has the form:

$$
\begin{aligned}
\int_{s}^{t} d \sigma_{1} \int_{s}^{\sigma_{1}} d \sigma_{2} \cdots \int_{s}^{\sigma_{n-1}} d \sigma_{n} \int d^{D} z_{1} \cdots d^{D} z_{n} \\
K_{t-\sigma_{1}}\left(x-z_{1}\right) \mathcal{R}_{\sigma_{1}, z_{1}} K_{\sigma_{1}-\sigma_{2}}\left(z_{1}-z_{2}\right) \cdots \mathcal{R}_{\sigma_{n}, z_{n}} K_{\sigma_{n}-s}\left(z_{n}-y\right),
\end{aligned}
$$

which decays esponentially in $|x-y|$ if $t>s$.

Notice that eq. (B.2) if valid for any smooth field $B_{\mu}(t, x)$. The Jacobian matrix $\bar{J}$ in eq. (2.6) coincides with $J$ when the field $B$ is replaced with the solution $\bar{B}_{t}$ of the flow equation. In order to generate the full perturbative expansion of $\bar{J}$, one needs to plug the perturbative expantion of $\bar{B}_{t}$ into the reminder $\mathcal{R}$ appearing in the integral equation (B.6), and solve it order by order. However provided that $\mathcal{R}$ is replaced by $\overline{\mathcal{R}}$, the structure of the generic term (B.9) of the expansion stays the same.

Open Access. This article is distributed under the terms of the Creative Commons Attribution License which permits any use, distribution and reproduction in any medium, provided the original author(s) and source are credited.

\section{References}

[1] S. Caracciolo, G. Curci, P. Menotti and A. Pelissetto, The energy momentum tensor for lattice gauge theories, Annals Phys. 197 (1990) 119 [INSPIRE].

[2] S. Caracciolo, G. Curci, P. Menotti and A. Pelissetto, The energy momentum tensor on the lattice: the scalar case, Nucl. Phys. B 309 (1988) 612 [INSPIRE].

[3] H. Suzuki, Energy-momentum tensor from the Yang-Mills gradient flow, Prog. Theor. Exp. Phys. 2013 (2013) 083B03 [arXiv: 1304.0533] [INSPIRE].

[4] H. Suzuki, Ferrara-Zumino supermultiplet and the energy-momentum tensor in the lattice formulation of $4 D \mathcal{N}=1 S Y M$, Nucl. Phys. B 868 (2013) 459 [arXiv:1209.2473] [InSPIRE].

[5] H. Suzuki, Remark on the energy-momentum tensor in the lattice formulation of $4 D \mathcal{N}=1$ SYM, Phys. Lett. B 719 (2013) 435 [arXiv:1209.5155] [INSPIRE].

[6] M. Lüscher, Trivializing maps, the Wilson flow and the HMC algorithm, Commun. Math. Phys. 293 (2010) 899 [arXiv:0907.5491] [INSPIRE].

[7] M. Lüscher, Properties and uses of the Wilson flow in lattice QCD, JHEP 08 (2010) 071 [arXiv: 1006.4518] [INSPIRE].

[8] M. Lüscher and P. Weisz, Perturbative analysis of the gradient flow in non-abelian gauge theories, JHEP 02 (2011) 051 [arXiv:1101.0963] [INSPIRE].

[9] M. Lüscher, Chiral symmetry and the Yang-Mills gradient flow, JHEP 04 (2013) 123 [arXiv:1302.5246] [INSPIRE]. 
[10] C.G. Callan Jr., Broken scale invariance in scalar field theory, Phys. Rev. D 2 (1970) 1541 [INSPIRE].

[11] K. Symanzik, Small distance behavior in field theory and power counting, Commun. Math. Phys. 18 (1970) 227 [InSPIRE].

[12] K. Symanzik, Small distance behavior analysis and Wilson expansion, Commun. Math. Phys. 23 (1971) 49 [InSPIRE].

[13] L. Giusti and H.B. Meyer, Thermal momentum distribution from path integrals with shifted boundary conditions, Phys. Rev. Lett. 106 (2011) 131601 [arXiv:1011.2727] [INSPIRE].

[14] L. Giusti and H.B. Meyer, Implications of Poincaré symmetry for thermal field theories in finite-volume, JHEP 01 (2013) 140 [arXiv:1211.6669] [INSPIRE].

[15] M. Lüscher and F. Palombi, Universality of the topological susceptibility in the SU(3) gauge theory, JHEP 09 (2010) 110 [arXiv: 1008.0732] [INSPIRE].

[16] M. Lüscher, Topology, the Wilson flow and the HMC algorithm, PoS(Lattice 2010)015 [arXiv: 1009.5877] [INSPIRE].

[17] P. Fritzsch and A. Ramos, The gradient flow coupling in the Schrödinger functional, JHEP 10 (2013) 008 [arXiv: 1301.4388] [InSPIRE].

[18] Z. Fodor, K. Holland, J. Kuti, D. Nogradi and C.H. Wong, The Yang-Mills gradient flow in finite volume, JHEP 11 (2012) 007 [arXiv:1208.1051] [INSPIRE].

[19] S. Borsányi et al., Anisotropy tuning with the Wilson flow, arXiv:1205.0781 [INSPIRE].

[20] R. Jackiw, Gauge covariant conformal transformations, Phys. Rev. Lett. 41 (1978) 1635 [INSPIRE].

[21] B.A. Berg, The transformations of nonAbelian gauge fields under translations, hep-th/0006045 [INSPIRE].

[22] C.G. Callan Jr., S.R. Coleman and R. Jackiw, A new improved energy-momentum tensor, Annals Phys. 59 (1970) 42 [INSPIRE].

[23] S.L. Adler, J.C. Collins and A. Duncan, Energy-momentum-tensor trace anomaly in spin 1/2 quantum electrodynamics, Phys. Rev. D 15 (1977) 1712 [INSPIRE].

[24] S.R. Coleman and R. Jackiw, Why dilatation generators do not generate dilatations?, Annals Phys. 67 (1971) 552 [INSPIRE].

[25] J.C. Collins, A. Duncan and S.D. Joglekar, Trace and dilatation anomalies in gauge theories, Phys. Rev. D 16 (1977) 438 [inSPIRE].

[26] K. Fujikawa, Energy momentum tensor in quantum field theory, Phys. Rev. D 23 (1981) 2262 [INSPIRE].

[27] M. Bochicchio, L. Maiani, G. Martinelli, G.C. Rossi and M. Testa, Chiral symmetry on the lattice with Wilson fermions, Nucl. Phys. B 262 (1985) 331 [InSPIRE]. 